\title{
Avaliação de revestimento de cromo por sputtering como alternativa à galvanização
}

\author{
Evaluation of sputtering chromium coating \\ as a electroplating substitute
}

\author{
Mônia Aline Hunger Geiger ${ }^{1}$, Leonardo Felix Scheffel ${ }^{1}$, \\ Carlos Leonardo Pandolfo Carone ${ }^{1}$, Fernando Dal Pont Morisso ${ }^{1}$, \\ Sandra Raquel Kunst ${ }^{2}$, Jane Zoppas Ferreira ${ }^{2}$, \\ Cláudia Trindade Oliveira ${ }^{1}$
}

\footnotetext{
${ }^{1}$ ICET, Universidade Feevale, RS-239, 2755, Novo Hamburgo, RS, Brasil.

${ }^{2}$ LACOR,Universidade Federal do Rio Grande do Sul - UFRGS, Avenida Bento Gonçalves, 9500, Porto Alegre, RS, Brasil.

e-mail: moniageiger@gmail.com, scheffel1234@gmail.com, carloscarone@feevale.br, morisso@ feevale.br; tessaro.sandra@gmail.com,jane.zoppas@ufrgs.br, cto@feevale.br
}

\section{RESUMO}

A elevada resistência à corrosão dos revestimentos decorativos de cromo hexavalente obtidos nos processos de galvanoplastia é bem conhecida pela sua grande utilização e alta eficiência. Esse revestimento tem sido amplamente utilizado na indústria moveleira e de calçados, obtido por eletrodeposição em peças de zamac. No entanto, o processo galvânico apresenta alto grau poluidor devido à quantidade de efluentes geradas e à toxicidade dos produtos químicos utilizados no processo. Sabe-se que o cromo hexavalente é um agente cancerígeno e os resíduos associados são perigosos e difíceis de serem tratados. Neste contexto, o desenvolvimento de novas tecnologias tem visado à obtenção de processos resistentes à corrosão, ambientalmente corretos e economicamente viáveis. A tecnologia alternativa que pode ir ao encontro desta necessidade é o PVD - physical vapor deposition, com o processo de sputtering. Neste sentido, este estudo tem como objetivo avaliar e comparar o desempenho de revestimentos de cromo aplicados por sputtering com verniz em relação ao desempenho do revestimento de cromo eletrodepositado. Para tanto, os revestimentos foram aplicados em zamac e foram caracterizados morfologicamente, eletroquimicamente e físico-quimicamente. Os resultados mostraram que os acabamentos de cromo depositados por sputtering apresentam características equivalentes quanto ao desempenho eletroquímico se comparados ao revestimento cromado no processo convencional, podendo ser uma alternativa de substituição com âmbito sustentável.

Palavras-chave: galvanização, sputtering, cromo, resistência a corrosão.

\begin{abstract}
The high corrosion resistance of decorative hexavalent chromium coating, obtained thru electroplating processes, is well known for its wide usage and elevated efficiency. This coating has been used all throughout furniture and shoe's industries, obtained via electrodeposition in Zamac pieces. However, the galvanic process presents a high pollution degree, due to the amount of generated waste, and the toxicity of the chemicals used. It is well known the hexavalent chromium is a carcinogenic agent, and how its residues are dangerous and hard treat properly. Because of this, the development of new technologies is aiming towards ecofriendly, economically viable, with a high corrosion resistance. The alternate technology that might be heading in this direction is PVD (Physical Vapor Deposition), with the sputtering process. In this sense, this paper's main objective is to evaluate and compare the development of varnished sputtering applied chromium coatings, in relation to the performance of electrodeposited chromium coatings. For such, they were applied in Zamac and morphologically, electrochemically, and physicallychemically characterized. The results showed that the sputtering deposited chromium coatings presented similar characteristics to the conventional coating method, so it might be a suitable and clean alternative in those regards.
\end{abstract}


Keywords: electroplating, sputtering, chromium, corrosion resistance.

\section{INTRODUÇÃO}

O Zamac 5 (Z5) é uma liga composta por zinco, alumínio, magnésio e cobre comumente utilizada na produção de peças para calçados, puxadores de móveis e em alguns metais sanitários [1,2]. Entretanto, sua resistência à corrosão é inferior comparada a outras ligas, como o latão, por exemplo. Este fato deve-se às imperfeições da matriz metálica, como porosidades, inerentes do processo de injeção sob pressão. O fluxo rápido e turbulento de material para dentro do molde pode provocar a adsorção e retenção tanto de gases formados por reações de oxidação de desmoldantes, ou dos próprios elementos de liga, quanto de ar atmosférico. A retenção de gases é muito afetada pelas taxas de resfriamento e pela pressão aplicada. Se a pressão aplicada durante o processo de injeção não for suficiente, ou a taxa de resfriamento for muito alta, os gases formados/adsorvidos ficam aprisionados na matriz metálica [3]. Porém, mesmo com essas propriedades, o uso do Z5 é justificado pelo seu baixo custo quando comparado ao latão [4,5].

Atualmente, a eletrodeposição de cromo é o principal meio para aprimorar as propriedades físicas e químicas do Z5, aumentando sua resistência à corrosão, abrasão e dureza, além de melhorar suas propriedades decorativas [6]. O processo de deposição de cromo por eletrodeposição, apesar de ser bastante utilizado devido ao seu baixo custo e alta eficiência, é precursor de vapores cancerígenos, alta contaminação atmosférica e hídrica. Portanto, existe a necessidade da aplicação de processos ambientalmente corretos, como physical vapor deposition (PVD). Este processo produz revestimentos superficiais com boa aparência, alta resistência ao desgaste, baixo atrito e boa resistência à corrosão [7].

Nos processos de PVD, os átomos são transportados sob a forma de vapor, por meio de um vácuo ou baixa pressão gasosa (plasma), do ambiente para o substrato, onde eles se condensam. Os processos de PVD podem ser divididos em duas categorias: a evaporação a vácuo e o sputtering $[8,9,10]$.

A porosidade dos filmes metálicos depositados, muitas vezes, torna-se um fator limitante na utilização de sputtering. Por isso, revestimentos base e de acabamento, tais como os vernizes, tem sido usados para proteger o substrato da corrosão e proporcionar resistência à abrasão [8]. A cura UV aplicada nesses revestimentos apresenta menor resistência quando expostos a ambientes externos em comparação aos revestimentos com cura térmica. A degradação dos filmes por UV pode ocorrer devido a alterações químicas e físicas durante a exposição [11].

Assim, o objetivo deste trabalho é avaliar e comparar as propriedades de resistência à corrosão do revestimento de cromo depositado pelo processo PVD com posterior aplicação de diferentes vernizes protetores, em relação ao revestimento de cromo depositado por meio do processo de eletrodeposição, ambos sobre o substrato de zamac 5. Além disso, serão analisados dois diferentes processos de cura dos vernizes aplicados, sendo um deles cura por radiação ultravioleta (UV) e o outro cura térmica.

\section{MATERIAIS E MÉTODOS}

\subsection{Preparação da Superfície}

As amostras de zamac 5 apresentaram geometria circular com diâmetro de $40 \mathrm{~mm}$, espessura de $5 \mathrm{~mm}$ e $2385 \mathrm{~mm}^{2}$ de área total. As amostras foram preparadas pelo processo de vibro-acabamento, por um período de $4 \mathrm{~h}$, para a remoção de rebarbas e nivelameto da superfície e polidas [12]. Posteriormente as peças foram lavadas com tricloroetileno para remover as sujidades. Outra parte de igual número de amostras passou somente pelo processo de vibro-acabamento, sem polimento.

As amostras com e sem polimento foram desengraxadas quimicamente em uma solução contendo $5 \%$ de hidróxido de sódio e $5 \%$ de cianeto de sódio por imersão, com agitação. Depois de lavadas e secadas em centrífuga, as peças foram fixadas em dispositivos denominados satélites e colocadas em estufa por uma hora a $90^{\circ} \mathrm{C}$ para complementar a secagem realizada na centrífuga. Em seguida, as amostras foram envernizadas pelo processo de pistolas pressurizadas com o verniz base em um sistema semiautomatizado. Este verniz base auxilia no processo de nivelamento da superfície e confere o brilho necessário ao substrato.

Após o envernizamento, as peças foram colocadas em um sistema de rotação para alastramento do verniz, permanecendo por cerca de 10 minutos e, depois disso, foram curadas no sistema de cura UV.

Na Tabela 1 estão indicados os parâmetros de processo utilizados no verniz base com cura UV. 
Tabela 1: Dados do processo de aplicação do verniz base UV.

\begin{tabular}{l|l|l|l|l|l|l}
\hline VERNIZ & COMPOSIÇÃo & $\begin{array}{l}\text { VISCOSIDADE } \\
\text { (CP - 25:C) }\end{array}$ & $\begin{array}{l}\text { APLICA- } \\
\text { ÇÃO }\end{array}$ & $\begin{array}{l}\text { FLASH- } \\
\text { OFF } \\
\text { (MIN) }\end{array}$ & $\begin{array}{l}\text { INTENSIDADE } \\
\text { DE CURA } \\
\text { (MW/CM }{ }^{2}\end{array}$ & LÂMPADA \\
\hline Base UV & $\begin{array}{l}\text { Poliester acrila- } \\
\text { do }\end{array}$ & 37,13 & Pistola & 10 & $\begin{array}{l}\text { UVA 280,3 } \\
\text { UVB 229,6 } \\
\text { UVC 71,1 } \\
\text { UVV 244,9 }\end{array}$ & $\begin{array}{l}\text { Mercúrio de } \\
\text { média pressão }\end{array}$ \\
\hline
\end{tabular}

\subsection{Eletrodeposição de cromo}

Anterior à etapa de eletrodeposição de cromo, foi feita a limpeza das peças de zamac por meio de prétratamento químico, limpeza em ultrasom, desengraxe catódico em solução alcalina contendo tensoativos, ativação ácida e lavagens. O processo de eletrodeposição consistiu na imersão em banho de cobre alcalino, que confere proteção à corrosão, seguido de cobre ácido, para nivelamento e brilho das peças. Depois disso, as amostras foram imersas em banho de níquel, com a finalidade de auxiliar o brilho para conferir o aspecto decorativo, e após foram cromadas.

A Tabela 2 apresenta as condições operacionais utilizadas em cada etapa do processo da galvanoplastia. Após a eletrodeposição de cromo e lavagens, as peças foram secadas com ar pressurizado e estufa a $100{ }^{\circ} \mathrm{C}$.

Tabela 2: Condições operacionais de aplicação do revestimento de cromo eletrodepositado.

\begin{tabular}{|c|c|c|c|c|}
\hline BANHO & $\begin{array}{l}\text { COBRE ALCALI- } \\
\text { NO }\end{array}$ & COBRE ÁCIDO & NÍQUEL & CROMO \\
\hline Ânodo & Cobre & Cobre fosforoso & Níquel & Chumbo \\
\hline $\begin{array}{l}\text { Densidade de } \\
\text { corrente }\left(\mathrm{A} / \mathrm{dm}^{2}\right)\end{array}$ & 1 & 4 & 4 & 10 \\
\hline Tempo (min) & 30 & 20 & 25 & 2,5 \\
\hline Temp. $\left({ }^{\circ} \mathrm{C}\right)$ & $45-50$ & $18-28$ & $55-60$ & $43-45$ \\
\hline Agitação & Catódica & $\mathrm{Ar}$ & $\mathrm{Ar}$ & Não \\
\hline Eletrólitos & $\begin{array}{l}60 \mathrm{~g} / \mathrm{L} \mathrm{CuCN} \\
28 \mathrm{~g} / \mathrm{L} \mathrm{KCN} \text { Aditi- } \\
\text { vos orgânicos }\end{array}$ & $\begin{array}{l}220 \mathrm{~g} / \mathrm{L} \mathrm{CuSO}_{4} 80 \\
\mathrm{~g} / \mathrm{L} \mathrm{H}_{2} \mathrm{SO}_{4} \text { Aditi- } \\
\text { vos orgânicos }\end{array}$ & $\begin{array}{l}250 \mathrm{~g} / \mathrm{L} \mathrm{NiSO}_{4} \\
75 \mathrm{~g} / \mathrm{L} \mathrm{NiCl}_{2} \\
45 \mathrm{~g} / \mathrm{L} \mathrm{H}_{3} \mathrm{BO}_{3} \\
45 \mathrm{~g} / \mathrm{L} \mathrm{H}_{3} \mathrm{BO}_{3}\end{array}$ & $\begin{array}{l}250 \mathrm{~g} / \mathrm{L} \\
\mathrm{H}_{2} \mathrm{CrO}_{4} 1,25 \\
\mathrm{~g} / \mathrm{L} \mathrm{H}_{2} \mathrm{SO}_{4}\end{array}$ \\
\hline Após banho & $\begin{array}{l}\text { lavagens e ativação } \\
\text { ácida com } \mathrm{H}_{2} \mathrm{SO}_{4} \\
5 \%\end{array}$ & $\begin{array}{l}\text { lavagens e ativa- } \\
\text { ção }\end{array}$ & lavagens & lavagens \\
\hline
\end{tabular}

\subsection{Revestimento de Cr depositado por sputtering}

Após a aplicação do verniz base as amostras passaram pelo processo de sputtering para a deposição do Cr. O equipamento utilizado para o sputtering tem capacidade para 6 satélites e 2 alvos, modelo DGK-36, marca Kolzer. Os principais parâmetros de deposição estão apresentados na Tabela 3. 
Tabela 3: Parâmetros do processo de deposição de cromo por sputtering.

\begin{tabular}{l|l}
\hline PARÂMETRO & CROMO \\
\hline Tipo de Alvo utilizado & Cromo \\
\hline \% Pureza & $99,99 \% \mathrm{Cr}$ \\
\hline Distância entre o Alvo e as peças (mm) & 150 \\
\hline Voltagem nominal (V) & 1000 \\
\hline Amperagem Nominal (A) & 25 \\
\hline Gás de trabalho & Argônio \\
\hline Vazão de gás (centímetros cúbicos padrão por minuto) & 80 \\
\hline Pressão (mbar) & $6 \times 10^{-4}$ \\
\hline
\end{tabular}

Após a deposição por sputtering, foram aplicados dois tipos de verniz nas amostras, denominados verniz top UV e verniz top térmico. O verniz top UV apresenta composição Poliester uretânico acrilado com subsequente cura UV. O verniz top térmico apresenta composição Acrílico uretânico catalisado com isocianato alifático com subsequente cura térmica. As condições de operação destes processos podem ser verificadas nas Tabelas 4 e 5 .

Tabela 4: Variáveis do processo de aplicação do verniz top UV.

\begin{tabular}{l|l|l|l|l|l|l}
\hline VERNIZ & CoMPOSIÇÃo & $\begin{array}{l}\text { VISCOSIDADE } \\
\left(\mathbf{C P}-\mathbf{2 5}^{\circ} \mathbf{C}\right)\end{array}$ & APLICAÇÃo & $\begin{array}{l}\text { FLAH- } \\
\text { OFF } \\
\text { (MIN) }\end{array}$ & $\begin{array}{l}\text { INTENSIDADE } \\
\text { CURA } \\
\left.\text { (MW/CM }^{2}\right)\end{array}$ & LÂMPADA \\
\hline $\begin{array}{l}\text { Top } \\
\text { UV }\end{array}$ & $\begin{array}{l}\text { Poliester ure- } \\
\text { tânico acrilado }\end{array}$ & 32,29 & Pistola & 10 & $\begin{array}{l}\text { UVA 372,2 } \\
\text { UVB 320,4 } \\
\text { UVC 102,5 } \\
\text { UVV 327,5 }\end{array}$ & $\begin{array}{l}\text { Mercúrio } \\
\text { de média } \\
\text { pressão }\end{array}$ \\
\hline
\end{tabular}

Tabela 5: Variáveis do processo de aplicação do verniz top térmico.

\begin{tabular}{l|l|l|l|l|l|l}
\hline VERNIZ & COMPOSIÇÃo & $\begin{array}{l}\text { VISCOSIDADE } \\
\left(\mathbf{C P}-\mathbf{2 5}^{\circ} \mathbf{C}\right)\end{array}$ & APLICAÇÃo & $\begin{array}{l}\text { FLASH- } \\
\text { OFF } \\
\text { (MIN) }\end{array}$ & $\begin{array}{l}\text { TEMP. } \\
\left({ }^{\circ} \mathbf{C}\right)\end{array}$ & $\begin{array}{l}\text { TEMPO DE } \\
\text { ESTUFA }\end{array}$ \\
\hline $\begin{array}{l}\text { Top tér- } \\
\text { mico }\end{array}$ & $\begin{array}{l}\text { Acrílico uretâ- } \\
\text { nico (A) catali- } \\
\text { sado com isoci- } \\
\text { anato alifático } \\
\text { (B) } \\
\text { Proporção 2 } \\
\text { A:1B }\end{array}$ & 26,20 & Pistola & 10 & $80^{\circ} \mathrm{C}$ & 40 minutos \\
\hline
\end{tabular}

A Tabela 6 indica a nomenclatura das amostras de zamac de acordo com os revestimentos depositados. 
Tabela 6: Nomenclatura das amostras de zamac de acordo com os revestimentos depositados.

\begin{tabular}{l|l}
\hline REVESTIMENTO & $\begin{array}{l}\text { IDENTIFICAÇÃO } \\
\text { NOS ENSAIOS }\end{array}$ \\
\hline Zamac da liga Z5 polida sem revestimento & Zamac Z5 \\
\hline Cromo eletrodepositado & Cr ED \\
\hline Cromo depositado por sputtering sem verniz top & Cr PVD \\
\hline Cromo depositado por sputtering com verniz top curado por UV & Cr PVD UV \\
\hline Cromo depositado pelo processo de sputtering com verniz top com cura térmica & Cr PVD T \\
\hline
\end{tabular}

\subsection{Caracterização das amostras}

A análise de morfologia e espessura de camada foram realizadas utilizando Microscópio Eletrônico de Varredura (MEV) modelo JEOL-JSM 6510CV com resolução de 129 eV. As análises de composição química foram realizadas por meio de EDS (Energy Dispersive Spectroscopy), acoplado ao MEV, da marca Thermo Scientific, modelo Ultra Dry.

A resistência à corrosão dos revestimentos foi avaliada por meio de curvas de polarização potenciodinâmica, com uso de potenciostato Autolab PGSTAT302 com faixa de potencial de aproximadamente $10 \mathrm{~V}$ e o software NOVA 1.8 . As análises foram realizadas a temperatura ambiente $\left(23^{\circ} \mathrm{C}\right)$ com aplicação de varredura de potencial de $0,01 \mathrm{~V} / \mathrm{s}$ iniciando em $-0,2 \mathrm{~V}$ até $1 \mathrm{~V}$ com relação ao potencial de corrosão.

As medições foram realizadas em uma solução de $0,6 \mathrm{~mol} / \mathrm{L}$ de $\mathrm{NaCl} \mathrm{pH}$ 6,87 em uma célula de três eletrodos, em que o fio de platina era o contra-eletrodo, o eletrodo de calomelano saturado (SCE) o de referência e o eletrodo de trabalho, com área de $1 \mathrm{~cm}^{2}$.

A medida de rugosidade foi realizada utilizando o perfilômetro de contato Mitutoyo Surftest SJ301. A molhabilidade das superficies foi caracterizada utilizando o ensaio ângulo de contato pelo método da gota séssil com o auxílio do programa de análise de imagens Surftens 3.0. Todos os ensaios foram realizados em triplicata.

A resistência à corrosão dos revestimentos foi avaliada por meio de curvas de polarização potenciodinâmica, com uso de potenciostato Autolab PGSTAT302 com faixa de potencial de aproximadamente $10 \mathrm{~V}$ e o software NOVA 1.8 . As análises foram realizadas a temperatura ambiente $\left(23^{\circ} \mathrm{C}\right)$ com aplicação de varredura de potencial de $0,01 \mathrm{~V} / \mathrm{s}$ iniciando em $-0,2 \mathrm{~V}$ até $1 \mathrm{~V}$ com relação ao potencial de corrosão. As medidas de espectrocospia de impedância eletroquímica fornecem informações de simulação de um circuito elétrico identificando as reações de interface. Para tanto, foi utilizado um eletrodo de referência de calomelano saturado, um contra-eletrodo de platina, um eletrodo de trabalho. A faixa de frequência utilizada durante a impedância foi de $100 \mathrm{kHz}$ até $3 \mathrm{mHz}$ com sinal senoidal de $20 \mathrm{mV}$. As análises foram feitas em solução $0,6 \mathrm{M}$ de $\mathrm{NaCl}$ durante 96 horas, com análises a cada 24 horas. A área de medida exposta foi de $6,5 \mathrm{~cm}^{2} \pm 0,2 \mathrm{~cm}^{2}$.

As medições foram realizadas em uma solução de $0,6 \mathrm{~mol} / \mathrm{L}$ de $\mathrm{NaCl} \mathrm{pH}$ 6,87 em uma célula de três eletrodos, em que o fio de platina era o contra-eletrodo, o eletrodo de calomelano saturado (SCE) o de referência e o eletrodo de trabalho, com área de $1 \mathrm{~cm}^{2}$.

A medida de rugosidade foi realizada utilizando o perfilômetro de contato Mitutoyo Surftest SJ301. A molhabilidade das superficies foi caracterizada utilizando o ensaio ângulo de contato pelo método da gota séssil com o auxílio do programa de análise de imagens Surftens 3.0. Todos os ensaios foram realizados em triplicata.

\section{RESULTADOS E DISCUSSÕES}

A Figura 1 apresenta a seção transversal da amostra de zamac com revestimento obtido por eletrodeposição (Zamac Z5) com magnitudes de 220 x e 1000 x. 


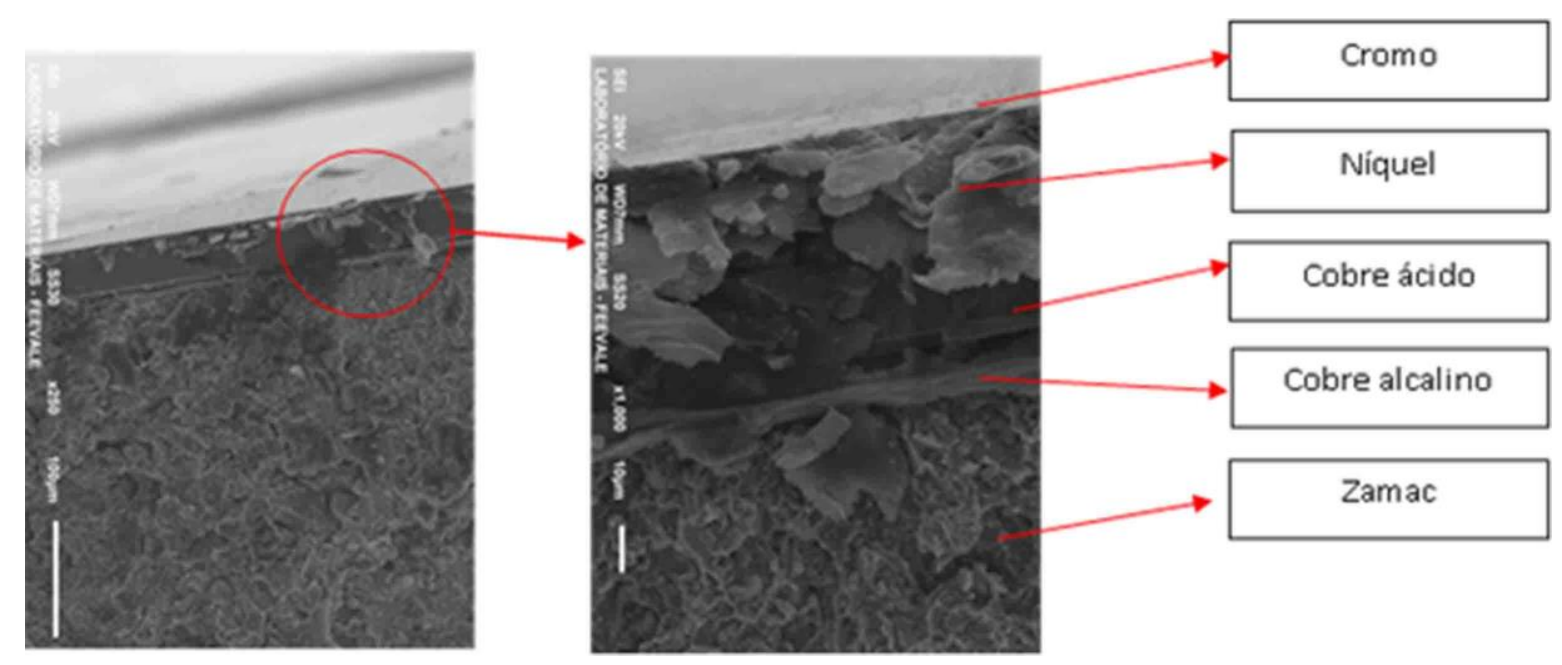

Figura 1: Seção transversal da amostra de zamac revestida com cromo eletrodepositado (Zamac Z5).

Observa-se que, no revestimento eletrodepositado, os filmes de cobre alcalino, cobre ácido, níquel e cromo apresentaram as seguintes médias de espessura: 8,97; 19,97; 24,92 e 0,267 $\mu \mathrm{m}$, respectivamente. As medidas de espessura de camada foram realizadas diretamente nas micrografias obtidas ao MEV.

De acordo com os parâmetros de densidade de corrente utilizados no processo produtivo, as espessuras de camada esperadas para o banho galvânico seriam de $10 \mu \mathrm{m}$ de cobre alcalino, $20 \mu \mathrm{m}$ de cobre proveniente do banho de cobre ácido, $25 \mu \mathrm{m}$ de níquel e cerca de $0,25 \mu \mathrm{m}$ de cromo. $\mathrm{O}$ banho de cobre alcalino cianídrico tem eficiência catódica de $75 \%$ e a deposição é de $1 \mu \mathrm{m}$ a cada 3 minutos, com aplicação de densidade de corrente de $1 \mathrm{~A} / \mathrm{dm}^{2}$. A eficiência catódica do banho de cobre ácido é de $100 \%$, o que corresponde a deposição de $1 \mu \mathrm{m}$ por minuto, com aplicação de densidade de corrente de $4 \mathrm{~A} / \mathrm{dm}^{2}$.

O banho de níquel possui eficiência de $95 \%$ e deposita cerca de $1 \mu \mathrm{m}$ por minuto, com aplicação de $4 \mathrm{~A} / \mathrm{dm}^{2}$ de densidade de corrente. Já o cromo tem eficiência catódica de apenas $15 \%$ e deposita cerca de $0,25 \mu \mathrm{m}$ a cada $2,5 \mathrm{~A} / \mathrm{dm}^{2}$ aplicado [13]. Isso indica que as espessuras estão de acordo com os valores esperados. A Figura 2 mostra as micrografias das seções transversais das peças de zamac com revestimento de cromo obtido por PVD, com posterior tratamento por UV e térmico. 

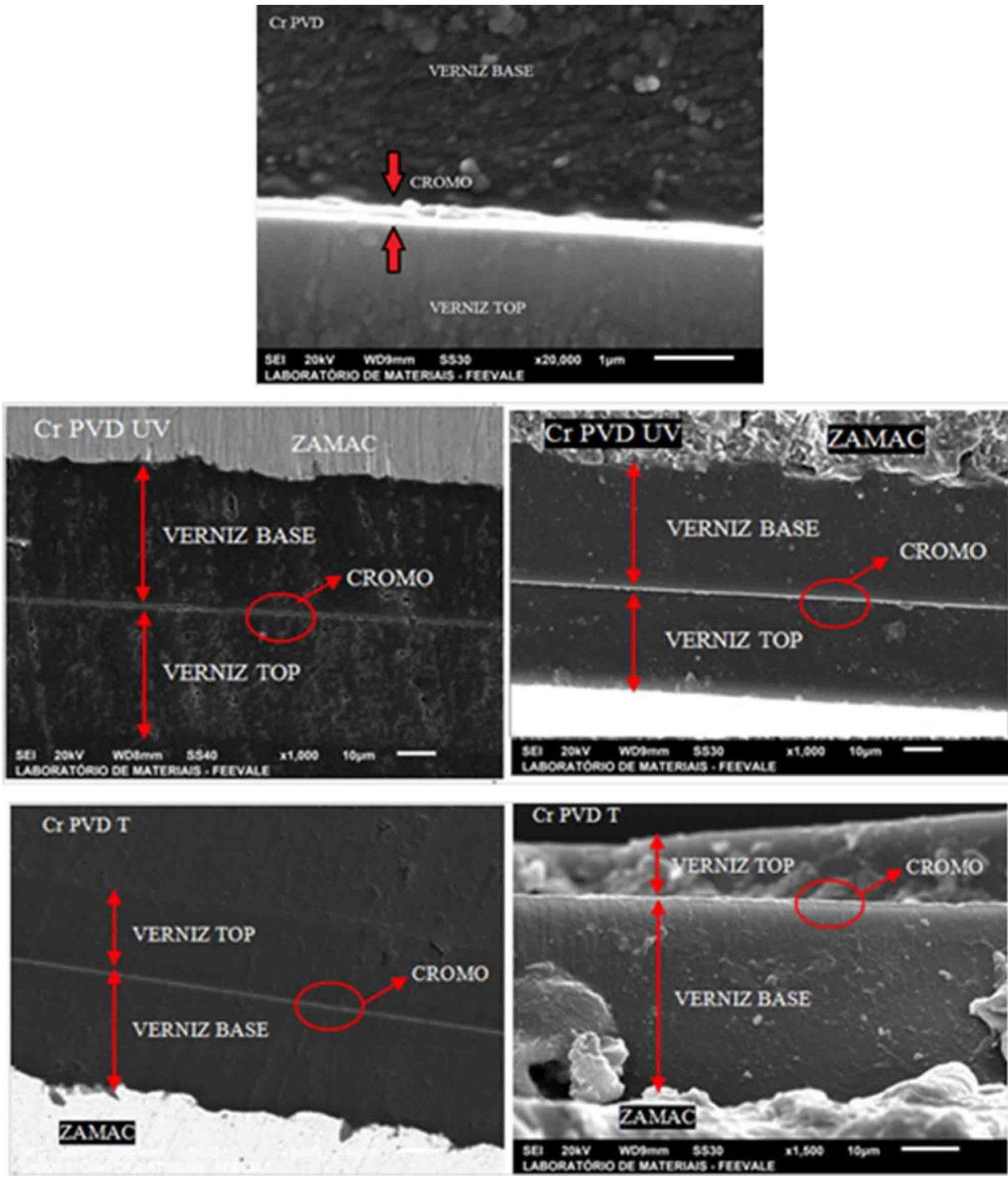

Figura 2: Micrografias das seções transversais das amostras (a) Cr PVD; (b) amostra Cr PVD UV embutida em resina; (c) amostra Cr PVD UV quebrada por imersão em nitrogênio; (d) amostra Cr PVD T embutida em resina; (e) amostra Cr PVD T quebrada por imersão em nitrogênio.

Observa-se na Figura 3b,c que o verniz base UV copia a superfície do zamac e nivela-a para receber os depósitos metálicos. Segundo Weber [14], o verniz base UV deve proporcionar uma superfície nivelada, de alto brilho, aderente ao substrato e auxiliar na proteção corrosão.

Depois da aplicação do metal por sputtering, com a superfície nivelada, observa-se que o verniz top apresenta uma espessura continua ao longo de toda a superfície (Figura 3 b,c,d,e). As espessuras das camadas de $\mathrm{Cr}$ depositadas por sputtering apresentaram média no valor de 0,219 $\mu \mathrm{m}$. As medidas de espessura de camada foram realizadas diretamente nas micrografias obtidas ao MEV.

A Tabela 7 apresenta os resultados encontrados para a espessura de camada dos sistemas verniz base e verniz top. A preparação destas amostras foi realizada por método de embutimento em resina epóxi e por quebra da amostra utilizando nitrogênio líquido. 
Tabela 7: Espessuras de camada de verniz aplicada $(\mu \mathrm{m})$.

\begin{tabular}{l|l|l|l|l}
\hline Amostra & Verniz & $\begin{array}{l}\text { Média por } \\
\text { embutimento }\end{array}$ & $\begin{array}{l}\text { Média por quebra } \\
\text { com N2 }\end{array}$ & Média Geral \\
\hline Cr PVD UV & Base & 34,823 & 30,919 & 32,871 \\
& Top & 32,548 & 26,313 & 29,431 \\
\hline Cr PVD T & Base & 28,053 & 32,873 & 30,463 \\
& Top & 20,632 & 11,776 & 16,204 \\
\hline
\end{tabular}

Observa-se que as espessuras de camadas são uniformes para os dois tipos de preparação, com exceção do verniz top no sistema Cr PVD T. Na Figura 2(e), observa-se que a camada não é uniforme. Como já citado anteriormente, as peças são fixas em satélites e pode haver regiões em que a cobertura é inferior, tais como as bordas superiores e inferiores das peças. Da mesma forma, o alastramento do verniz é muito importante para que a espessura de camada seja uniforme em toda a extensão da peça.

A Figura 3 apresenta as micrografias de topo dos sistemas Cr ED, Cr PVD UV, Cr PVD T. Na amostra de Cr ED, observa-se uma superfície homogênea, livre de poros. Já as amostras Cr PVD UV, Cr PVD T apresentaram um filme homogêneo e sem fissura e/ou microporos.

Na amostra de Cr PVD UV, percebe-se a presença de microporos que se estendem por todo o revestimento. A ocorrência desses poros podem estar relacionados com a presença de bolhas de ar em função da evaporação do solvente retido. O filme de cromo adere à camada polimérica (verniz curado por radiação ultravioleta), iniciando as reações de polimerização e reticulação entre os produtos químicos das espécies presentes [15]. Essas reações ocorrem através da ação da radiação UV, sendo necessário adicionar à composição química uma substância sensível a essa radiação: o fotoiniciador. Ele é a molécula responsável pela absorção da radiação UV, na qual o mecanismo de cura inicia a reação de polimerização do radical livre. Para que essa reação ocorra, a luz ultravioleta deve penetrar no revestimento, para que o fotoiniciador possa absorve-la. Para tanto, duas condições são necessárias: a luz ultravioleta deve ter energia suficiente para penetrar no material e o revestimento não deve ser opaco, para não interferir na absorção dessa radiação [16].

O processo pelo qual uma substância líquida curada por UV é convertido em revestimento sólido após exposição à radiação UV pode ser dividida em quatro etapas: (1) interação entre luz UV e o fotoiniciador; (2) o rearranjo químico do fotoiniciador resultando na formação de radicais livres; (3) a reação desses radicais livres com a insaturação dos monômeros chamado de solvente reativo, iniciando as reações de polimerização por adição; (4) a continuação da polimerização dos radicais livres através da insaturação dos monômeros resultando no acoplamento com o polímero (tinta por cura UV), para que as interações resultem em excelente adesão no substrato [15]. As duas primeiras fases (interações do fotoiniciador com a luz UV e a formação dos radicais livres) pode ocorrer de acordo com os mecanismos de fragmentação homolítica do iniciador ou subtração de hidrogênio [17]. Na Figura 3 observa-se a presença de bolhas de ar em oriundas da evaporação do solvente retido, que consiste no solvente reativo que não foi totalmente polimerizado.
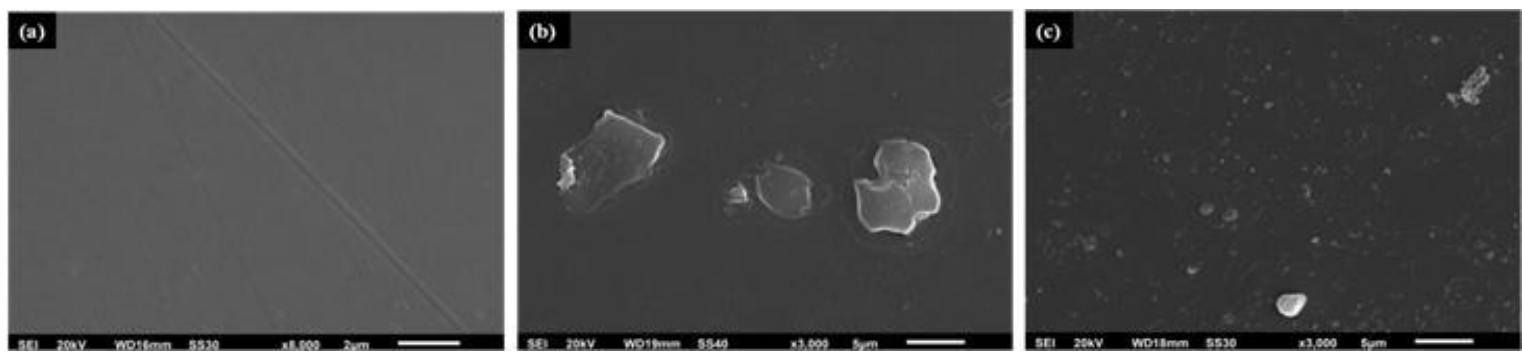

Figura 3: Micrografias da região de topo das amostras de zamac com revestimentos de: (a) Cr ED, (b) Cr PVD UV e (c) Cr PVD T. 
A Figura 4 apresenta os resultados dos ensaios de polarização do Zamac Z5, CrPVDUV, Cr PVDT e Cr PVD.

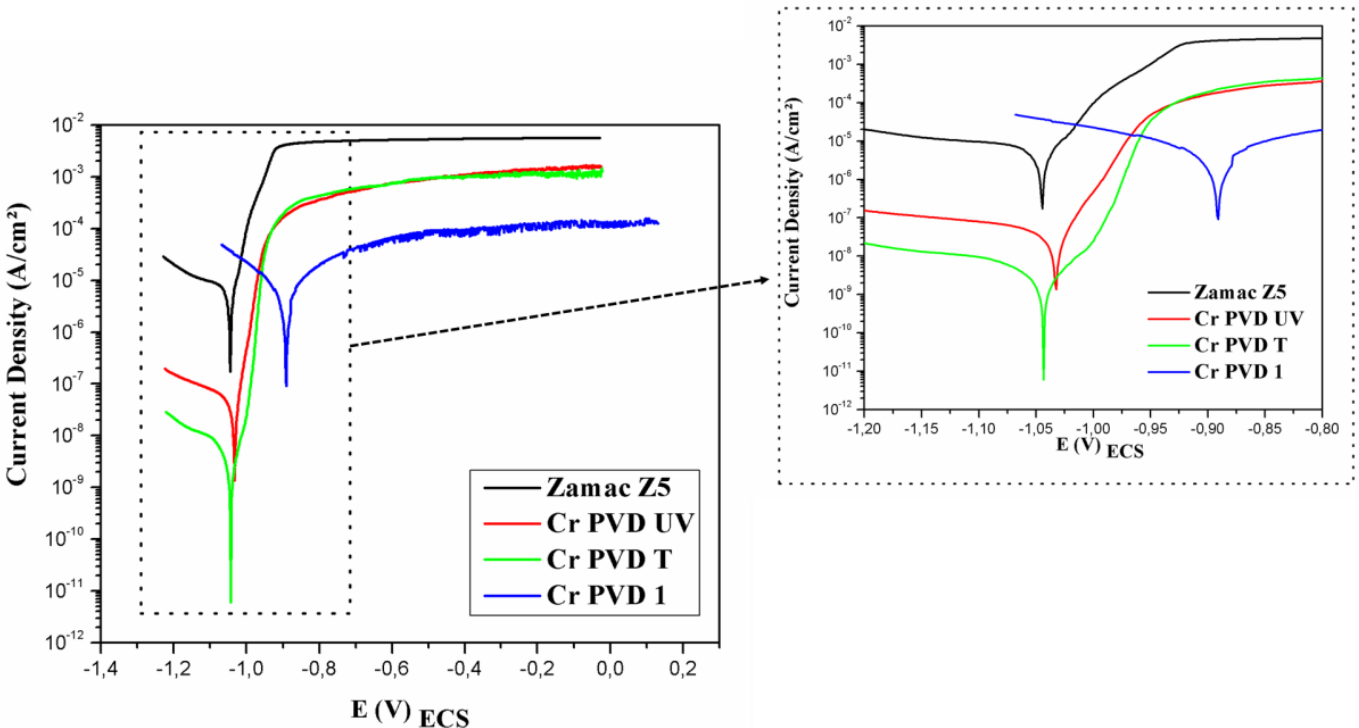

Figura 4: Curvas de polarização de amostras de Zamac Z5, Cr PVD 1, Cr PVD UV, Cr PVD T e Cr PVD em $\mathrm{NaCl}$ $0,6 \mathrm{M}, \mathrm{pH}$ 6,87 em 1 hora de imersão.

Na Figura 4, observa-se que todos os revestimentos apresentam uma região anódica e uma região catódica bem definida, com potencial de corrosão aproximadamente constante para quase todos os revestimentos, incluindo o Zamac Z5. Além disso, todos os revestimentos com depósito de cromo com verniz mostraram corrente de corrosão e densidade de corrente limite inferior ao do Zamac Z5. Isso indica que os revestimentos promoveram a formação de uma barreira efetiva entre o substrato e o meio, protegendo efetivamente o substrato do ambiente corrosivo. A extrapolação das retas de Taffel estão apresentados na Tabela 8 com os valores de resistência à corrosão do Zamac Z5 e os revestimentos aplicados, os quais podem ser comparados.

Tabela 8: Parâmetros eletroquímicos de corrosão de todos os sistemas estudados.

\begin{tabular}{|c|c|c|c|c|}
\hline AMOSTRA & $\begin{array}{l}\text { E CORROSÃO (V) } \\
\text { CALCULADO }\end{array}$ & $\begin{array}{l}\text { I CORROSÃO } \\
\left(\mu \mathrm{A} / \mathrm{CM}^{2}\right)\end{array}$ & $\begin{array}{l}\text { TAXA DE CORROSÃO } \\
\text { (MM/ANO) }\end{array}$ & $\begin{array}{l}\text { RESISTÊNCIA DE } \\
\text { POLARIZAÇÃO (K } \Omega)\end{array}$ \\
\hline Zamac Z5 & $-1,039$ & 15,663 & 0,499890 & 0,777 \\
\hline Cr ED 1 & $-1,028$ & 0,285 & 0,006826 & 43,750 \\
\hline $\mathrm{Cr}$ ED 2 & $-1,018$ & 6,377 & 0,151810 & 4,874 \\
\hline Cr ED 3 & $-0,763$ & 0,211 & 0,005060 & 151,780 \\
\hline Cr ED 4 & $-0,342$ & 0,247 & 0,006009 & 125,670 \\
\hline Cr PVD 1 & $-0,902$ & 65,756 & 1,575100 & 5,657 \\
\hline Cr PVD 2 & $-0,395$ & 0,031 & 0,000740 & 1008,900 \\
\hline Cr PVD UV & $-1,035$ & 0,034 & 0,000818 & 262,620 \\
\hline Cr PVD T & $-1,044$ & 0,002 & 0,000052 & 6018,400 \\
\hline
\end{tabular}


De acordo com a Tabela 8, o potencial de corrosão encontrado para o Zamac Z5 foi de -1,04V. Este valor está de acordo com os resultados de outros estudos em Zamac 5, em que os ensaios de OCP e curvas de polarização em $\mathrm{NaCl} 0,6 \mathrm{M}$ mostraram potencial de corrosão entre -1 e $-1,02566 \mathrm{~V}$ [4,5]. Esse comportamento pode estar relacionado com a reação de redução do oxigênio $\left(\mathrm{O}_{2}+2 \mathrm{H}_{2} \mathrm{O}+4 \mathrm{e}-\rightarrow 4 \mathrm{OH}-\right)$, a qual é a principal reação catódica de corrosão do zinco neste meio. Observa-se que os valores de potencial de corrosão encontrados para o Zamac Z5 são muito próximos aos do zinco puro (-1,06V) e zinco eletrodepositado (-1,02), o que indica que o comportamento da liga frente à corrosão segue os mesmos padrões de comportamento do zinco [18].

Ainda com relação ao Zamac Z5, pode-se observar um aumento de densidade de corrente a partir do potencial de corrosão característico da formação de produto de corrosão sem apresentar uma região passiva bem definida. Inicialmente há dissolução do metal, juntamente com polarização por concentração. A dissolução continua dando início à formação de uma camada de produtos de corrosão sobre a superfície. Por fim, a curva apresenta um aumento de potencial associado a uma estabilidade da densidade de corrente, chegando à corrente limite e formação de camada pseudo-passiva, não totalmente passiva pois não há decaimento da densidade de corrente [5]

Com relação aos revestimentos de Cr ED 3 e 4, apesar de apresentarem comportamento distinto entre os revestimentos, a densidade de corrente de corrosão foi menor e resistência à polarização maior do que o Zamac Z5. No sistema Cr ED 3 houve um aumento considerável na inclinação anódica e estabilização da corrente numa região que pode ser indicada como pseudo-passiva, que inicialmente bloqueia a dissolução dos revestimentos. Este efeito pode estar associado com a resposta de um óxido não-condutor

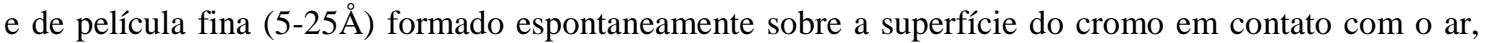
composto de hidróxido de cromo não cristalino na parte exterior com ilhas de $\mathrm{Cr}_{2} \mathrm{O}_{3}$ cristalino disperso na parte interna. Esta região passiva se estende até potenciais mais altos e depois é seguido por um aumento acentuado na densidade de corrente, comportamento este relacionado com a ruptura local da película de óxido protetor e crescimento de um pite estável. Este revestimento teve uma baixa corrente de corrosão pela formação da barreira anódica e uma inibição catódica de reação de redução de oxigênio, ambos atribuídos ao efeito protetor contínuo da película de óxido/hidróxido de cromo formada nas superfícies [19].

$\mathrm{Na}$ amostra $\mathrm{Cr}$ ED 4 pode-se perceber, na curva anódica, um aumento da corrente com o potencial, correspondendo a um mecanismo de corrosão uniforme, provavelmente relacionado à dissolução do metal [20].

O revestimento de Cr PVD não exibiu microfissuras, mas sim defeitos de porosidade e vazios comuns para o processo de PVD. O potencial de corrosão do revestimento de Cr PVD foi de - 0,075 V, mais nobre que o $\mathrm{Cr} \mathrm{ED}$, que apresentou potencial de $-0,703 \mathrm{~V}$. Este resultado demonstrou que o revestimento obtido por PVD foi mais resistente à corrosão, potencialmente devido ao filme de óxido passivo [19].

Para o revestimento Cr PVD 1, que não foi aplicado verniz, observou-se maior densidade de corrente de corrosão, seguido do Zamac Z5 e da amostra Cr ED2.

Para as amostras contendo verniz, Cr PDV UV e Cr PVD T, observaram-se as menores densidades de corrente e menores taxas de corrosão, bem como maior resistência à polarização, o que indica um comportamento melhor com respeito à corrosão em comparação ao revestimento de cromo eletrodepositado. Percebe-se que o verniz inibe a oxidação do cromo quando aplicado logo após a deposição de sputtering. Além disso, este comportamento ainda foi melhor para o verniz térmico, em comparação ao UV, apresentando uma resistência de polarização de três ordens de grandeza comparativamente a duas ordens de grandeza do sistema curado por UV [21].

As Figura 5, 6 e 7 apresentam os resultados dos ensaios de espectroscopia de impedância eletroquímica (EIE) do Zamac Z5, CrPVDUV, Cr PVDT, CrED e Cr PVD em 1 hora, 72 horas e 168 horas respectivamente em $\mathrm{NaCl} 0,6 \mathrm{M}$.

De acordo com os diagramas de Bode (Figura 5), em 1 hora de imersão em $\mathrm{NaCl}$ 0,6 M, observa-se um fenômeno em alta frequência e com maior ângulo de fase para a amostra CrPVDT em comparação as demais. Esta constante de tempo está associada à barreira física formada pelo verniz poliuretânico (Acrílico uretânico catalisado com isocianato alifático). Os poliisocianatos alifáticos conferem às tintas poliuretânicas excelentes propriedades de resistência à intempérie, pois esses isocianatos são resistentes à ação do ultravioleta. Esses vernizes também se caracterizam por uma excelente estabilidade da cor, aderência e resistência à corrosão [21]. Estes resultados corroboram com os resultados de polarização apresentados na Figura 4 para a amostra CrPVDT, a qual apresentou a menor densidade de corrente de corrosão e a maior resistência de polarização quando imersa também em $\mathrm{NaCl}$ 0,6 M. No entanto, esta amostra apresenta um 
fenômeno em baixa frequência com ângulo de fase em torno de $60^{\circ}$ associado ao processo corrosivo, o que foi identificado por Cao et al. [22] como mudança na cinética de corrosão do controle de transferência de carga para controle por difusão.
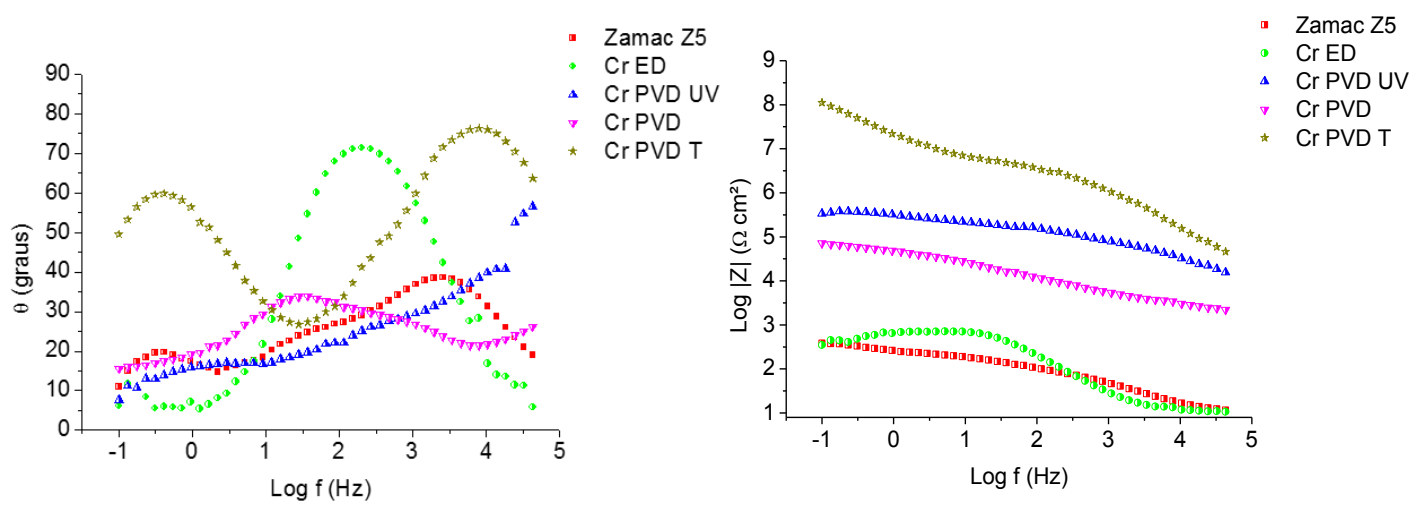

Figura 5: Espectros de impedância eletroquímica, Bode: (a) ângulo de fase e (b) módulo log /z/, para todas as amostras estudadas em 1 hora de imersão em $\mathrm{NaCl} 0,6 \mathrm{M}$.

Para a amostra CrPVDUV também se observa um fenômeno em alta frequência associado ao filme barreira formado na interface metal/eletrólito, porém, com menor ângulo de fase quando comparado a amostra CrPVDT (Figura 5-a). Observa-se que o verniz inibe a oxidação do cromo quando aplicado logo após a deposição por sputtering. Comparadno com a amostra CrPVDUV, a amostra CrPVDT apresenta maior resistência à corrosão (Figura 5-b), o que também está de acordo com os resultados de polarização em que a amostra CrPVDT apresentou uma resistência de polarização duas ordens de grandeza maior que a amostra CrPVDUV.

A amostra CrPVD apresentou um fenômeno em média frequência com baixo ângulo de fase o que está associado à permeabilidade do eletrólito no revestimento por sputtering. Uma justificativa para o baixo desempenho corrosivo seria que nesta amostra não foi aplicado o verniz, tornando-a susceptível à corrosão. Apesar da amostra CrPVD não exibir microfissuras, ela apresenta defeitos de porosidade e vazios comuns no processo de PVD. Já a amostra CrED apresentou apenas um fenômeno em média fre-

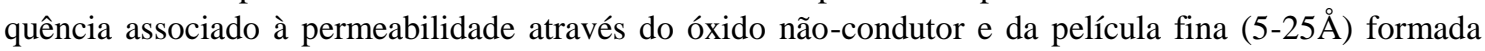
espontaneamente sobre a superfície do cromo em contato com o ar [19].

O Zamac Z5, em 1 hora de imersão, apresentou três constantes de tempo, em alta, média e baixa frequência. $\mathrm{O}$ fenômeno em alta frequência pode ser associado ao óxido de alumínio formado instantaneamente ao ar. De acordo com Liu et al. [23], a primeira constante de tempo em alta frequência para o zamac refere-se às propriedades da camada do produto de corrosão, que neste caso é devido ao $\mathrm{Al}_{2} \mathrm{O}_{3}$ formado ao ar. $\mathrm{O}$ fenômeno em média frequência está associado à permeabilidade do eletrólito através da camada de óxido e a constante em baixa frequência pode estar associado ao processo corrosivo que ocorre na interface do produto de corrosão com a liga. Neste caso, a constante em baixa frequência pode estar associada à baixa espessura do óxido formado ao ar.
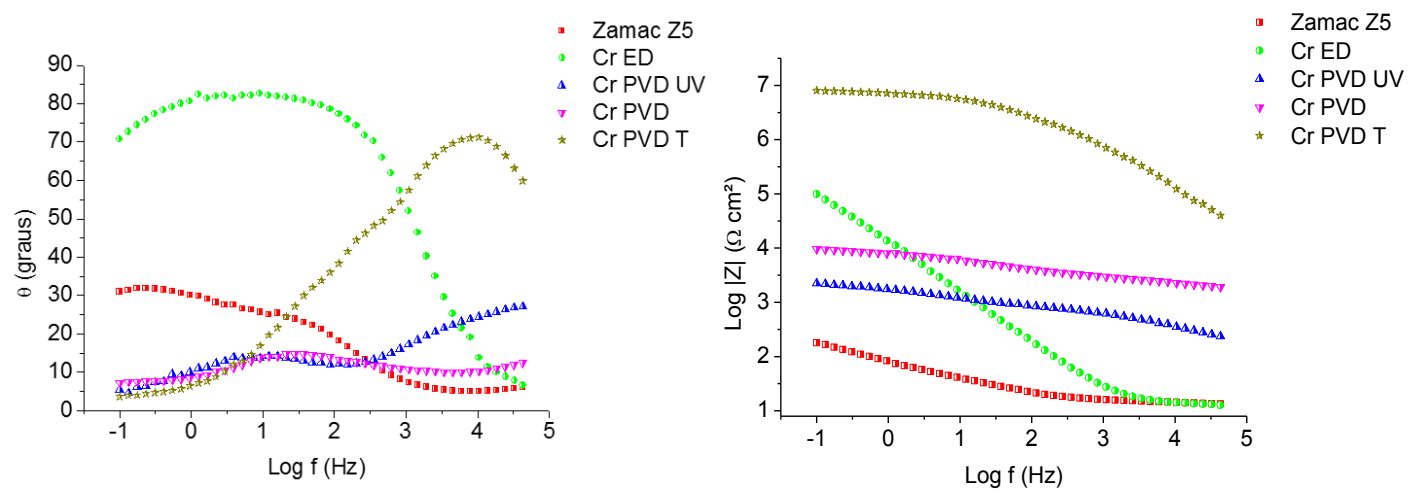

Figura 6: Espectros de impedância eletroquímica, Bode: (a) ângulo de fase e (b) módulo log /z/, para todas as amostras estudadas em 72 horas de imersão em $\mathrm{NaCl} 0,6 \mathrm{M}$. 
Em 72 horas de imersão (Figura 6-a) para a amostra CRPVDT, observa-se apenas o fenômeno em alta frequência com ângulo de fase similar ao processo eletroquímico em 1 hora de imersão (Figura 5-a) o que implica que a constante de tempo associado a barreira do verniz resiste à 72 horas de imersão e consequentemente apresenta uma estabilidade na resistência a corrosão do verniz térmico (acrílico uretânico catalisado com isocianato alifático) o que está associado as características de resistência a corrosão dos poliisocianatos alifáticos que conferem às tintas poliuretânicas.

Para a amostra CrPVDUV o fenômeno em alta frequência permanece, o que indica que o verniz apresenta resistência química devido as interações do fotoiniciador com a luz UV e a formação dos radicais livres em que ocorreu de acordo com os mecanismos de fragmentação homolítica do iniciador ou subtração de hidrogênio [17]. No entanto, se observa-se um fenômeno em média frequência associado a permeabilidade através do verniz UV e fez com que a resistência total da amostra diminuísse (Figura 6-b). Esta constante pode estar associada a presença de microporos que se estendem por todo o revestimento conforme observado nas micrografias ao MEV (Figura 3). A ocorrência desses poros podem estar relacionados com a presença de bolhas de ar em função da evaporação do solvente retido.

Para a amostra CrPVD observa-se que o fenômeno em média frequência permanece (Figura 6-a), porém, com o menor ângulo de fase quando comparado ao diagrama de impedância de 1 hora de imersão (Figura 5-a). Este menor ângulo de fase indica maior permeabilidade do eletrólito no revestimento por sputtering não resistindo a longos tempos de imersão devido a esta amostra não apresentar o verniz que inibe a oxidação do cromo quando aplicado logo após a deposição de sputtering. Já para a amostra CrED o fenômeno em média frequência se desloca para baixa frequência devido ao processo corrosivo devido a transferência de carga para controle por difusão.

No Zamac Z5 observa-se que o aumento do tempo de imersão (72 horas), o fenômeno em alta frequência desaparece e surge o fenômeno em média frequência e o fenômeno em baixa frequência aumenta o ângulo de fase. De acordo com Cao et al. [22] a constante em alta frequência desaparece pois esta constante de tempo corresponde às propriedades do produto de corrosão, o que está relacionado com o óxido formado, o que é bastante fino e com espessura na ordem de nanômetros, portanto sem propriedades protetivas quando exposto ao $\mathrm{NaCl}$ por tempos prolongados de imersão. Já o fenômeno em média frequência, aumenta a permeabilidade transformando-se em produto de corrosão, o que pode ser visto com o aumento do fenômeno em baixa frequência.
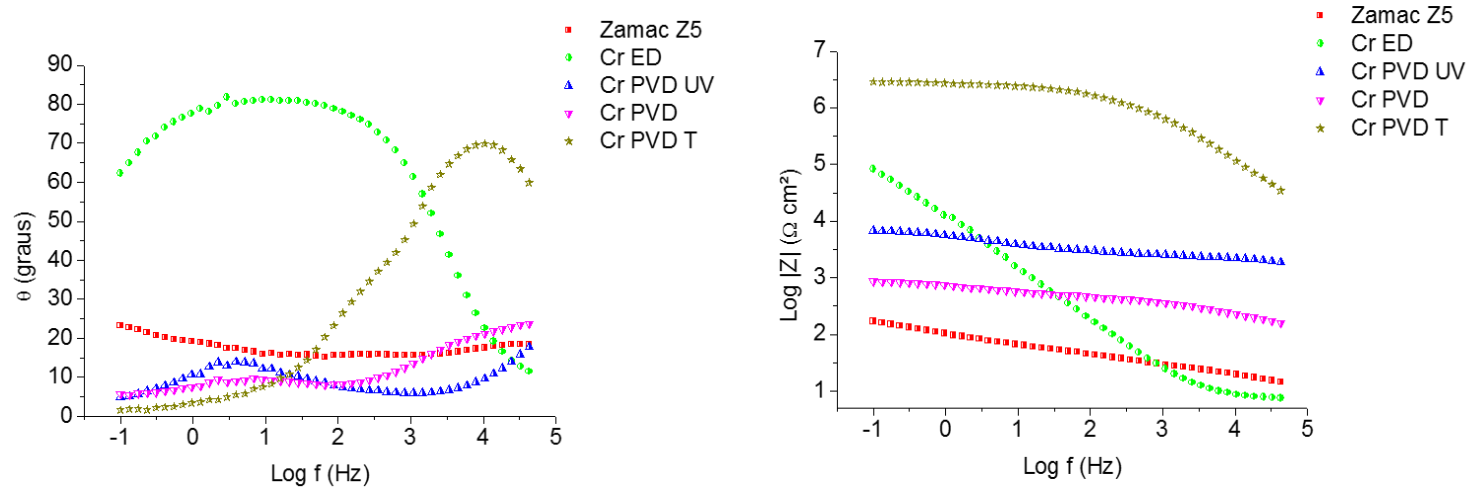

Figura 7: Espectros de impedância eletroquímica, Bode: (a) ângulo de fase e (b) módulo log /z/, para todas as amostras estudadas em 168 horas de imersão em $\mathrm{NaCl} 0,6 \mathrm{M}$.

Em 168 horas de imersão observa-se que o fenômeno em alta frequência associado a barreira física do verniz permanece para a amostra Cr PVD T (Figura 7-a) denotando a resistência à corrosão deste sistema devido ao verniz poliuretânico catalisado com isocianato alifático e que resiste à longos tempos de imersão em $\mathrm{NaCl}$ 0,6 M como pode ser observado na Figura 7-b em que esta amostra apresentou a maior resistência do módulo de impedância após 168 horas de imersão entre todas as amostras estudadas.

Para a amostra Cr PVD UV observa-se que o fenômeno em alta frequência desaparece (Figura 7a) associado ao filme formado com o verniz curado por radiação UV e apenas nota-se uma constante de tempo em média frequência associado a permeabilidade do eletrólito através do verniz o que indica que este verniz não resiste à longos tempos de imersão (Figura 7-b) e isso pode estar associado ao ajuste de formulação do verniz curado por radiação UV sendo necessário a utilização de monômeros (diluentes 
reativos) tri funcionais ou tetra funcionais a fim de melhorar a resistência química do verniz, no entanto, pode ter como consequência a perda de aderência [17].

Já a amostra Cr PVD observa-se que o fenômeno em média frequência observado em 72 horas de imersão (Figura 6-a) é deslocado para alta frequência em 168 horas de imersão (Figura 7-a), mas, permanecendo com baixo ângulo de fase. De acordo com Cao et al. [22] a constante em alta frequência corresponde às propriedades do produto, o que está relacionado ao óxido formado, que é bastante fino, portanto sem propriedades protetivas quando exposto a $\mathrm{NaCl}$.

Para a amostra CrED o fenômeno em média frequência deslocado para baixa frequência permanece em 168 horas de imersão (Figura 7-a) devido ao processo corrosivo devido a transferência de carga para controle por difusão. Este efeito pode estar associado com a resposta de um óxido não-condutor e de

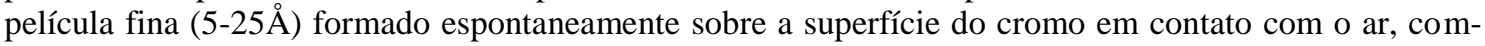
posto de hidróxido de cromo não cristalino na parte exterior com ilhas de $\mathrm{Cr}_{2} \mathrm{O}_{3}$ cristalino disperso na parte interna. Esta região passiva se estende até potenciais mais altos e depois é seguido por um aumento acentuado na densidade de corrente, comportamento este relacionado com a ruptura local da película de óxido protetor e crescimento de um pite estável.

Na amostra de Zamac Z5 observa-se um fenômeno mais acentuado em baixa frequência (Figura 7a) o que se caracteriza pela ocorrência do processo corrosivo na interface produto de corrosão/liga metálica, portanto, os valores de impedância diminuem com o tempo de imersão e consequentemente diminuindo a resistência à corrosão de acordo com o espectro de módulo de impedância (Figura 7-b).

Outra característica que influencia na resistência à corrosão é o grau de porosidade dos revestimentos, o qual tem sido calculado quantitativamente. Utilizando as técnicas eletroquímicas é possível estimar a porosidade dos revestimentos, partindo das curvas de polarização [24,25]. Os percentuais de porosidade dos revestimentos de cromo eletrodepositado e de cromo depositado por sputtering com e sem verniz foram calculados a partir da Equação 1 e podem ser observados na Tabela 9.

$$
P=R p s / R p 10^{-\Delta E \operatorname{Corr} / \beta a}
$$

Em que:

P é o total de porosidade do revestimento, Rps é a resistência de polarização do substrato, Rp é a resistência de polarização do substrato revestido, $\Delta$ ecorr é a diferença entre o potencial de corrosão do substrato revestido e o substrato e $\beta$ a é o declive anódico de Taffel.

Pode ser observado que o revestimento Cr PVD T apresenta o menor percentual de porosidade e o revestimento $\mathrm{Cr}$ ED 2 apresenta o maior percentual de porosidade.

Tabela 9: Porosidade estimada dos revestimentos estudados.

\begin{tabular}{l|l}
\hline AMOSTRA & \% POROSIDADE \\
\hline Cr PVD T & 0,00013 \\
\hline Cr PVD 2 & 0,00075 \\
\hline Cr PVD UV & 0,00296 \\
\hline Cr ED 3 & 0,00505 \\
\hline Cr ED 4 & 0,00598 \\
\hline Cr ED 1 & 0,01775 \\
\hline Cr PVD 1 & 0,13643 \\
\hline Cr ED 2 & 0,15924 \\
\hline
\end{tabular}

A Figura 9 apresenta o gráfico com as medidas de ângulo de contato obtidas para todas as amostras estudadas. Os revestimentos Cr ED e Cr PVD UV apresentaram valores de ângulo de contato próximos, o que indica que nestas amostras a molhabilidade é semelhante. Uma correlação é normalmente ob- 
servada entre a molhabilidade de um dado material e seu desempenho anticorrosivo. Os materiais com maior caráter hidrofóbico são tipicamente mais resistentes contra a corrosão em ambientes aquosos [20].

Os revestimentos Cr PVD UV a base de monômeros poliéster uretânico acrilado curados por radiação UV são produtos de reação de um diisocianto com compostos hidroxilados (conforme Figura 8). A partir dessa reação, observa-se um aumento do peso molecular e, por consequência, do uretano acrilado. Uma das consequências desse processo é a diminuição de periculosidade do isocianato devido à diminuição da volatilidade do composto que contém isocianato [15]. Uma outra vantagem é que se pode obter revestimentos com propriedades anti-corrosivas e consequentemente com maior hidrofobicidade (Figura 9), o que corrobora com os resultados obtidos nas curvas de polarização (Figura 4).

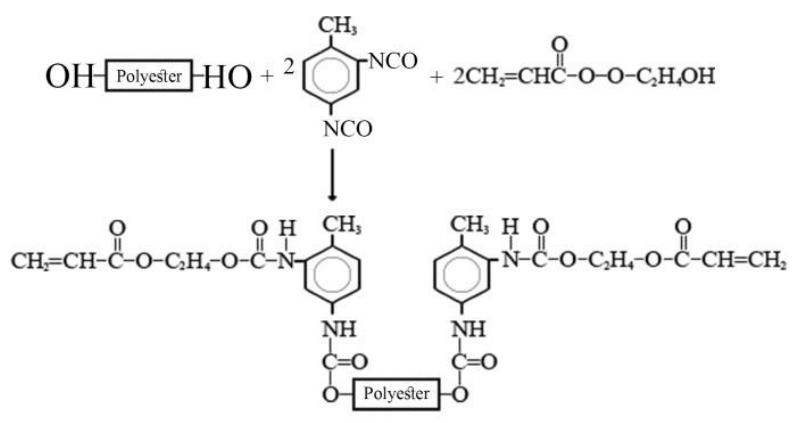

Figura 8: Reação de um diisocianto com compostos hridroxilados para a formação de um poliéster uretano acrilado.

O revestimento Cr PVD T apresentou o menor valor para ângulo de contato e, consequentemente, teria a maior molhabilidade. Contudo, este comportamento observado para o verniz térmico (acrílico uretânico catalisado com isocianato alifático) obtido pelo processo de PVD pode estar associado a dois principais fatores [7,26] que podem causar a variação do molhamento denominada histerese do ângulo de contato são: não homogeneidade topográfica causada pela rugosidade do substrato ou porosidade superficial e a não homogeneidade química devido à presença de contaminantes, segregação e inclusões na superfície do sólido.

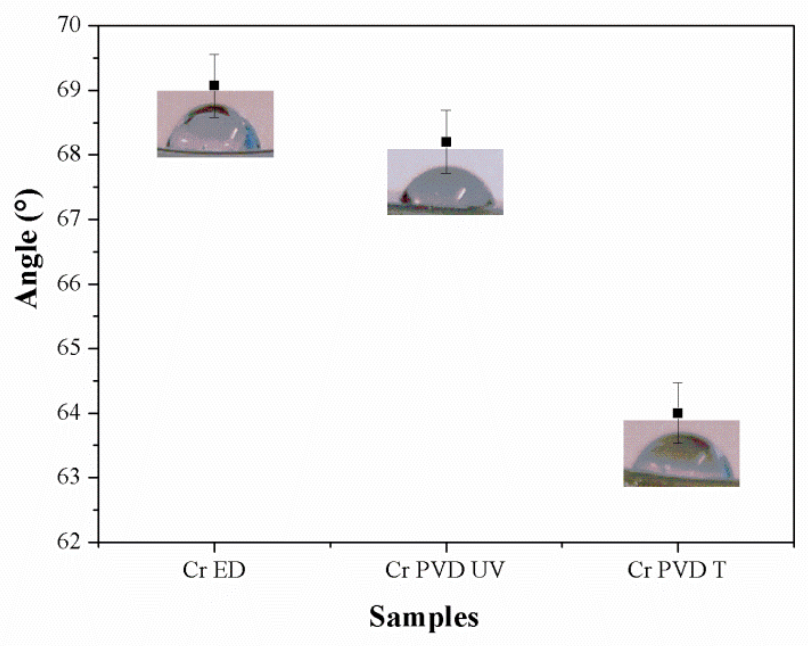

Figura 9: Gráfico dos valores de ângulo de contato para as amostras.

\section{CONCLUSÕES}

Os testes de caracterização apresentaram melhor desempenho para os revestimentos obtidos por sputtering com aplicação de verniz em comparação ao revestimento eletrodepositado, sendo o verniz top térmico acrílico uretânico catalisado aplicado sobre o cromo que mostrou o melhor resultado.

Na caracterização morfológica, os sistemas com depósito de cromo apresentaram uma espessura e filmes homogêneos. Contudo, nos ensaios eletroquímicos, para os sistemas Cr ED e Cr PVD o potencial 
de corrosão se deslocou para valores mais positivos, indicando aumento na resistência a corrosão. O sistema Cr PVD T, apresentou menor taxa de corrosão e menor porosidade, indicando sua maior desempenho anticorrosivo.

No entanto, o revestimento Cr PVD T apresentou o menor valor para ângulo de contato e, consequentemente, teria a maior molhabilidade, este resultado foi associado a variação do molhamento denominada histerese do ângulo de contato devido a porosidade superficial e a não homogeneidade química relacionado à presença de contaminantes, segregação e inclusões na superfície do revestimento.

Observou-se que o verniz poliéster acrilado por cura UV não apresentou um desempenho satisfatório e necessita de ajustes em sua formulação. Seria interessante utilizar esse mecanismo de cura uma vez que o processo de cura dos vernizes UV é muito mais rápido se comparado ao processo de cura térmica em estufa.

Os resultados obtidos indicam que estes revestimentos podem ser uma alternativa viável e sustentável para substituição do cromo galvânico nas aplicações avaliadas.

\section{BIBLIOGRAFIA}

[1] FAN, H., CHEN, C.H.Z, HE, Q. et al., "Effects of the casting temperature on microstructure and mechanical properties of the squeeze-cast $\mathrm{Al}-\mathrm{Zn}-\mathrm{Mg}-\mathrm{Cu}$ alloy", Journal of Alloys and Compounds, v. 504, n.2, pp. 42-45, 2010.

[2] CHEN, W.K., BAI, C.Y., LIU, C.M. et al., "The effect of chromic sulfate concentration and immersion time on the structures and anticorrosive performance of the $\mathrm{Cr}$ (III) conversion coatings on aluminum alloys", Applied Surface Science, v. 256, n.16, pp. 4924-4929, 2010.

[3] GHOMASHCHI, M.R., VIKHROV, A. "Squeeze casting: Overview", Journal of Materials Processing Technology, v. 101, n.1-3, pp. 1-9, 2000.

[4] FÜHR, L.T., LUDWIG, G.A., MARTINS, M.R., et al., "Effects of mould temperature in equeeze casting of zamak 5”, Materials Science Forum, v. 775/776, pp. 729-732, 2014.

[5] MOUANGA, M., BERÇOT, P., RAUCH.J, Y., "Comparison of corrosion behavior of zinc in $\mathrm{NaCl}$ and in $\mathrm{NaOH}$ solutions, Part I: Corrosion layer characterization”, Corrosion Science, v. 52, pp. 3984-3992, 2010.

[6] SNYDER, D.L., "Decorative Chromium Plating Basics. Metal Finishing", Universal metal finishing handbook, New York: D. Hopwood, v. 110, n.9A, pp. 293-303, 2012.

[7] CHIPATECUA, Y.L., OLAYA, J.J., ARIAS, D.F. “Corrosion behaviour of CrN/Cr multilayers on stainless steel deposited by unbalanced magnetron sputtering”, Vacuum, v. 86, n.9, pp. 1393-1401, 2012.

[8] MATTOX, D.M. Handbook of physical vapor deposition (PVD) Processing. British Library, Boston, William Andrew, 2010.

[9] KELLY, P., ARNELL, R.D. "Magnetron Sputtering: A Review of Recent Developments and Applications”, Vacuum, v. 56, n.3, pp. 159-172, 2000.

[10] KOLEV, I. Technology designed for a wide range of solutions. Hauzer Techno Coating BV- n 20. Venlo. NETHERLAND, 2012.

[11] RUIZ, C.S.B., MACHADO, L.D.B., VOLPONI, J.E. et al., "Influence of sample composition and processing parameters on the UV cure of clear coatings", Nuclear Instruments and Methods in Physics Research Section B: Beam Interactions with Materials and Atoms, v. 208, pp. 309-313, 2003.

[12] DAVIS, J.R. Metals Hanbook: Properties and Selection. 2.ed. Editora ASM. 2010.

[13] CASTRO, E.P., SILLOS, R.M. Manual Técnico Tratamento de Superfícies. São Bernardo do Campo SP: Surtec do Brasil Ltda, 4. ed, 2012.

[14] WEBER, E., ABELL, P., UV_“Curable Basecoats and Clearcoats for Automotive Lighting”, Radtech Report, Nov, Dec. 2003.

[15] FAZENDA, J.M.R., Tintas \& Vernizes Ciência e Tecnologia. São Paulo, Abrafati, pp. 822-838, 2010.

[16] LINA. "FDA Compliant Testing for Energy Curable Materials Used in Flexible Packaging. RadTech Europe 2003", RadTech Europe: The Hague, The Netherlands, v. 1, pp. 323-335, Berlin, Germany, 2003.

[17] MELBY, E.G., HARMAN, P., WEAVER, J., et al., "Novel Adhesives and Coatings for Food 
Packaging with Low Extractables”, UV \& EB Conference, RadTech International: Bethesda, MD, 2003.

[18] LI, M.C., JIANG, L.L., ZHANG, Q.W. et al., "Electrochemical corrosion behavior of nanocrystalline zinc coatings in 3.5\% NaCl solutions", Journal of Solid State Electrochemistry, v. 11, pp. 1319-1325, 2007.

[19] MAENG, S., AXE, L., TYSON, T.A. et al., "Corrosion behaviour of electrodeposited and sputtered Cr coatings and sputtered Ta coatings with $\alpha$ and $\beta$ phases", Surface and Coatings Technology, v. 200, pp. 5767-5777, 2006.

[20] IMAZ, N., OSTRA, M., VIDAL, M. et al., "Corrosion behaviour of chromium coatings obtained by direct and reverse pulse plating electrodeposition in $\mathrm{NaCl}$ aqueous solution”, Corrosion Science, v. 78, pp. 251-259, 2014.

[21] STUDER, K., DECKER, C., BECK, E. et al., "Thermal and photochemical curing of isocyanate and acrylate functionalized oligomers", European Polymer Journal, v. 41, pp. 157-167, 2005.

[22] CAO, Z., CHE, C., WANG, Y. et al., "Influence of Nd addition on the corrosion behavior of zn5\% Al alloy in 3,5wt\% NaCl solution, Surface Science, v. 426, pp. 67-76, 2017.

[23] LIU, J.C., ZHANG, G., NOGI, M. et al. "The role of Zn precipitates and Cl- anions in pitting corrosion of Sn-Zn solderalloys. Corrosion Science, v. 92, pp. 263-271, 2015.

[24] JAFARZADEH, S., ADHIKARI, A., SUNDELL, P.E. et al., "Study of PANI-MeSA conducting polymer dispersed in UV-curing polyester acrylate on galvanized steel as corrosion protection coating", Progress in Organic Coatings, v. 70, n. 2-3, pp. 108-115, 2011.

[25] AHN, S.H., YOO, J.H., CHOI, Y.S. et al. "Corrosion behavior of PVD-grown WC-(TilyxAlx)N films in 3.5\% NaCl solution”, Surface and Coatings Technologiy, v. 162, pp. 212-221, 2003.

[26] DA LUZ, A.P., RIBEIRO, S., PANDOLFELLI, V.C. "Artigo revisão: uso da molhabilidade na investigação do comportamento de corrosão de materiais refratários”, Ceramica, v. 54, n. 330, pp. 174-183, 2008.

\section{ORCID}

Mônia Aline Hunger Geiger Leonardo Felix Scheffel

Carlos Leonardo Pandolfo Carone

Fernando Dal Pont Morisso

Sandra Raquel Kunst

Jane Zoppas Ferreira

Cláudia Trindade Oliveira
https://orcid.org/0000-0001-8018-461X

https://orcid.org/0000-0002-3502-7225

https://orcid.org/0000-0002-4084-4502

https://orcid.org/0000-0002-9653-9857

http://orcid.org/0000-0002-8060-3981

https://orcid.org/0000-0002-3137-297X

https://orcid.org/0000-0002-4472-5359 